Original Article

\title{
The Effect of Ultrasound-Assisted Debridement Combined with Vacuum Pump Therapy in Deep Sternal Wound Infections
}

\author{
Lachmandath Tewarie, MD, ${ }^{*}$ Nikolay Chernigov, MD, ${ }^{*}$ Andreas Goetzenich, MD, PhD,
} Ajay Moza, MD, Rüdiger Autschbach, MD, PhD, and Rashad Zayat, MD

\begin{abstract}
Purpose: To assess whether a combined treatment of low-frequency ( $25 \mathrm{kHz})$ ultrasonicdebridement systems followed by vacuum-assisted wound closure (VAC) produces a better outcome in deep sternal wound infections (SWIs) compared to that of VAC alone.

Methods: We evaluated 45 consecutive patients ( 25 males) between January 2013 and December 2016, in whom deep SWI was treated with a combination of low-frequency ultrasonic debridement system followed by vacuum-assisted closure (group $\mathbf{A}, \mathbf{n}=\mathbf{2 3}$ ) or with only vacuum-assisted closure therapy (group $B, n=22$ ). Our final step in both groups was a secondary wound closure with a musculocutaneous flap.

Results: In both groups, a similar variety of bacteria were isolated. The time between eradication and secondary wound closure was significantly shorter in group $A$ (7.3 \pm 4.8 vs. 19.9 \pm 17.2 days, $p=0.001$ ). After a third debridement session, $95.7 \%$ of microbiological cultures were negative in group A versus $54.5 \%$ in $B(p=0.001)$. Duration of antibiotic treatment $(p=0.003)$ and hospitalization time $(p=0.0001)$ were significantly shorter in group A.

Conclusion: The use of low-frequency ultrasonic debridement system is an effective, less invasive technique to combat wound infection. In combination with vacuum-assisted closure therapy, we documented good mid-term results in our patients.
\end{abstract}

Keywords: cardiac surgery, postoperative complications, sternal wound infection, ultrasonicassisted wound debridement, vacuum-assisted wound closure

\section{Introduction}

Sternal wound infections (SWIs) are one of the most common complications after cardiac surgery. Its incidence

Department of Thoracic and Cardiovascular Surgery, RWTH University Hospital, Aachen, Germany

Received: December 18, 2017; Accepted: January 17, 2018

Corresponding author: Rashad Zayat, MD. Department of Thoracic and Cardiovascular Surgery, RWTH University Hospital, Pauwelsstr. 30, 52074 Aachen, Germany

Email: rzayat@ukaachen.de

(C2018 The Editorial Committee of Annals of Thoracic and Cardiovascular Surgery. This work is licensed under a Creative Commons Attribution-NonCommercial-NonDerivatives International License.

*Both authors contributed equally to this study.

${ }^{+}$Abstract was presented at the 31st EACTS Annual meeting in Vienna, Austria. varies between $1 \%$ and $3 \%$ (extremes, $0.5 \%-10 \%$ ). ${ }^{1)}$ SWIs are associated with high postoperative mortality and morbidity, also significantly reducing quality of life, increasing length of hospital stay, and hospital readmission rates. ${ }^{2,3)}$

Adequate wound-bed preparation, including tissue debridement and infection control play a crucial role in the treatment of wound disorders. ${ }^{4)}$ Negative pressure wound therapy, also known as vacuum-assisted wound closure (VAC), has traditionally been used worldwide in patients with chronic complicated non-healing wounds since 1997.5) Widespread biofilms have been observed in chronic and acute wounds. Established biofilms have an enhanced tolerance and resistance to antimicrobial interventions. Microbes growing within a biofilm have been reported to be up to 1000 times more tolerant to antimicrobials than their planktonic counterparts. ${ }^{4)}$ 
Low-frequency ultrasound-assisted wound (LF-UAW) debridement system is a debridement modality that provides bacterial biofilm destruction, preferentially removes necrotic issue, decreases bacterial counts, minimizes blood loss, and is relatively painless. $\left.{ }^{6}\right)$ An additional point is that the ultrasound waves at the wound surface promote separation and sloughing of necrotic material without damaging the unaltered surrounding tissue.7) The use of LF-UAW has not yet been widely used in SWI, and its relevance and effectiveness as an alternative method remains unknown. Our hypothesis is that using a LF-UAW (low frequency, $25 \mathrm{kHz}$ ) in addition to VAC therapy will completely remove the bacterial biofilms, and preserve more vital sternal tissue compared to VAC therapy alone in patients with deep sternal wound infection (DSWI) without mediastinitis. In addition to surgical advantages, we hypothesized that this technique will reduce hospital costs by shortening the length of the hospital stay and the length of antimicrobial therapy.

\section{Materials and Methods}

\section{Patients and data collection}

Between January 2013 and December 2016, we performed 4273 median sternotomies for cardiac surgery procedures in our institution. In all, 45 (1.1\%) patients developed DSWI. All patients who developed DSWI after an uncomplicated primary cardiac procedure were eligible for inclusion in the study. Exclusion criteria were as follows: patients with a sternocutaneous fistula with mediastinal infection, patients with mediastinitis, patients with bacteremia and multiple organ failure, and patients with a percutaneous or surgical tracheotomy. From January 2013, we began with LF-UAW therapy in our department. In this retrospective study, we compared all patients with DSWI who were treated either with a LF-UAW and VAC (group A, N = 23) or with surgical debridement and VAC alone (group $\mathrm{B}, \mathrm{N}=22$ ). Two attending cardiac surgeons were trained to use the LF-UAW therapy in our institution, and began to treat all their DSWI with the LF-FUAW, at the same time the rest of our attending cardiac surgeons continued to treat DSWI patients with the gold standard, convectional VAC. Due to these circumstances, we had two different DSWI groups (groups A and B) from 2013 to 2016.

The following data were retrospectively collected from our department's database: demographics, preoperative risk factors, perioperative and postoperative parameters, intubation time, microbiological findings from sternal wound, occurrence of SWI, duration of antimicrobial therapy, and all-cause in-hospital mortality.

All patients with DSWI were monitored for at least 6 months during their time in our outpatient department.

\section{Wound infection diagnosis and definition}

A SWI was diagnosed by a clinical examination (signs of local infection, drainage of pus, fistulas, and fever), computed tomography (CT) scans (retrosternal fluid collection and sternal dehiscence) and lab findings (leukocytosis and C-reactive protein). In all cases, the diagnosis was confirmed by microbiological findings. Microbiological wound cultures were taken prior to wound debridement. Two sets of wound swabs were sent for immediate Gram stain and culture. In cases with negative wound culture, wound tissues (soft or bone) were collected for polymerase chain reaction (PCR) assay. Once a wound disorder became evident, appropriate antibiotics were administered based on culture and sensitivity results. Prior to the debridement of the wound, informed consent was obtained. General anesthesia was practiced in all patients.

\section{Wound infection treatment strategies}

The LF-UAW system (Sonoca 185, Söring Inc. Germany) consists of a generator and an ultrasonic transducer (Fig. 1) ${ }^{8)}$ Normal saline $(0.9 \% \mathrm{NaCl})$ was used as connecting medium for ultrasound waves to the tissues and was procured by a transducer-attached line. The transducer and a foot pedal were connected to the generator. The low frequency $(25 \mathrm{kHz})$ allows for great depth of penetration with only minimal thermal stress to the tissue. The high intensity $\left(35-40 \mathrm{~W} / \mathrm{cm}^{2}\right)$, on the other hand, creates a strong cavitation effect, which is essential for the therapeutic effect. Cavitation is defined as the formation of micro-bubbles in a liquid medium induced by ultrasound-induced compression/traction forces. This technique allows the cleaning of the wound of necrotic tissue while suppressing the development of surface microflora. At this power level, exposure to the ultrasound is not destructive to healthy tissues, selectively removing only pathologically altered tissue. Without causing extra tissue damage, necrotic wound tissue is separated from the vital tissue. With a force setting between $60 \%$ and $100 \%$ of the device's maximal power, the wound surface is treated for $15-30$ seconds per $\mathrm{cm}^{2}$ as recommended by the manufacturer. All foreign material and infected scar tissue in and around the wound disorder 

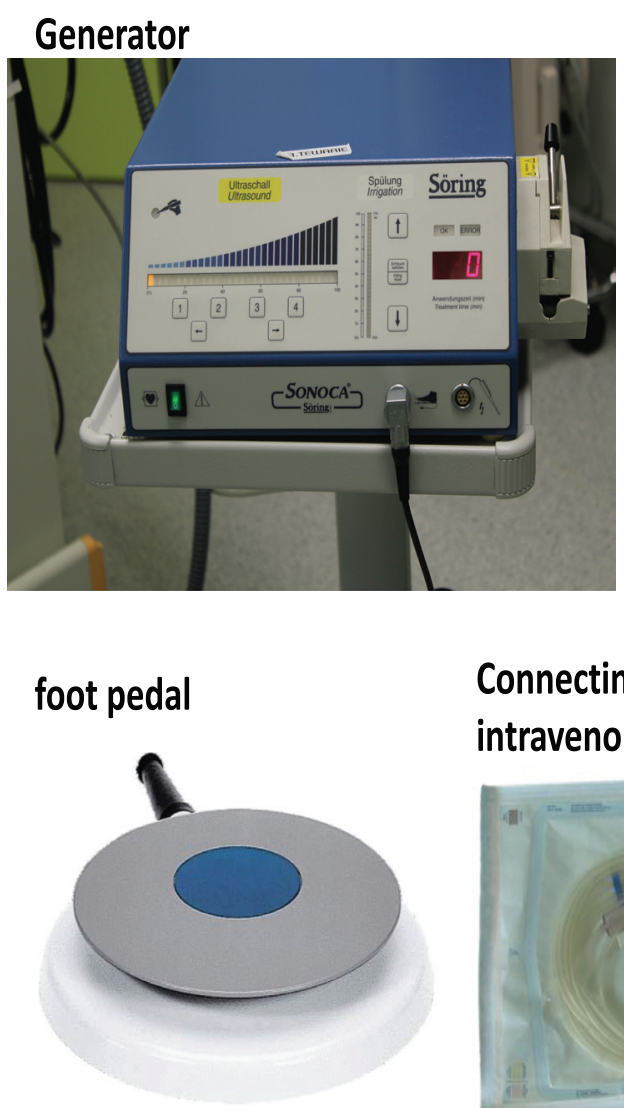

\section{Connecting cables and intravenous tubing}

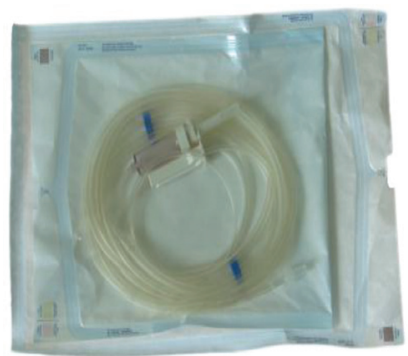

saline $(0.9 \% \mathrm{NaCl})$

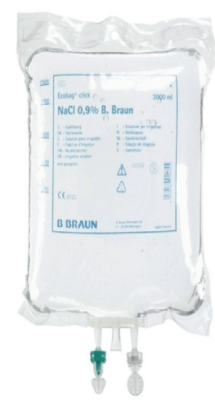

Fig. 1 The ultrasound-assisted wound system (Sonoca 185, Söring Inc. Germany).

area were removed with standard surgical instruments. LF-UAW was used to debride the wound, cleansing the surrounding vital tissue, followed by VAC, using the VAC (Lohmann \& Rauscher International GmbH \& Co. Germany). The LF-UAW/VAC debridement consists of multiple sessions in 3-day intervals (Fig. 2).

In group $\mathrm{B}$, the conventional surgical wound debridement consisted of the excision of the necrotic and devascularized tissue, removing foreign bodies followed by VAC therapy. VAC was continued in 3-day intervals, until the wound appeared macroscopically clean, granulation tissue had formed and microbiology results revealed no remaining bacterial contamination. Both treatment VAC and LF-UAW were performed under general anesthesia in the operating room. After each debridement session, patients in both groups were admitted to the general ward. When the wound appeared macroscopically clean and after achieving two consecutive sterile microbiological findings from the DSWI, VAC and LF-UAW were then terminated. Our final step in both groups was secondary wound closure with musculocutaneous flap. All patients underwent a similar preoperative assessment and a variety of cardiac procedures using standard median sternotomy. All patients undergoing cardiac surgery in our department routinely receive Mupirocin nasal ointment on the day before operation and a single shot of perioperative prophylactic antibiotics with either ampicillin/sulbactam (Unacid (Sultamicillintosilat-Dihydrat), Pfizer Pharma PFE GmbH, Berlin, Germany), or if a patient has a penicillin allergy, clindamycin.

\section{Statistical analysis}

All data are represented as the percent of the patient's group, while the absolute numbers are shown in percentages, or as the mean plus or minus standard deviation. The handling of statistical data was performed in SPS 22 (SPSS, IBM Corporation, Armonk, NY, USA). Student's t-test was performed where the normal distribution of data was estimated. Otherwise, a Mann-Whitney test was performed. A $p$ value of $<0.05$ was considered statistically significant.

\section{Results}

The incidence of DSWI in our study was $1.1 \%$. The mean time until the DSWI occurred was $26.9 \pm 18.1$ 
(A)

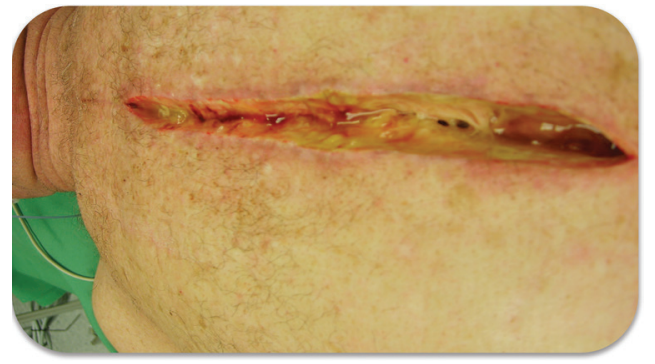

(C)

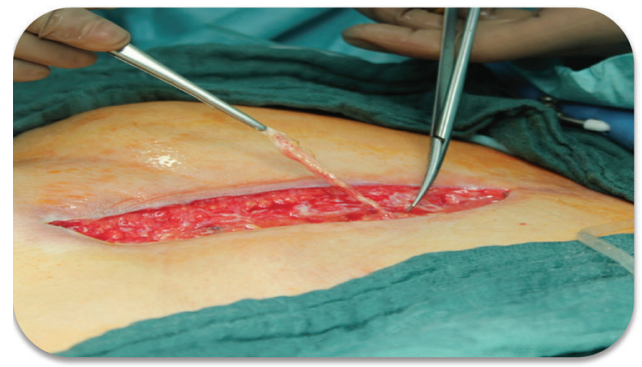

(B)

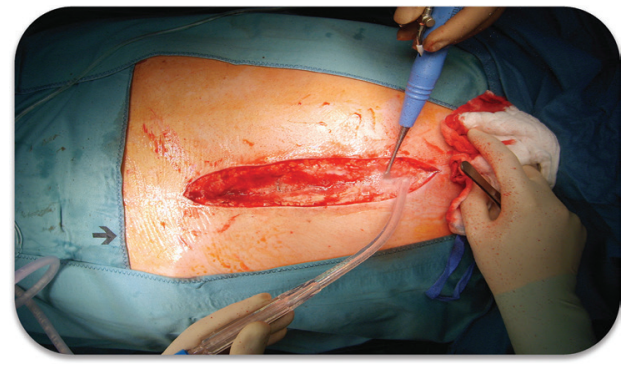

(D)

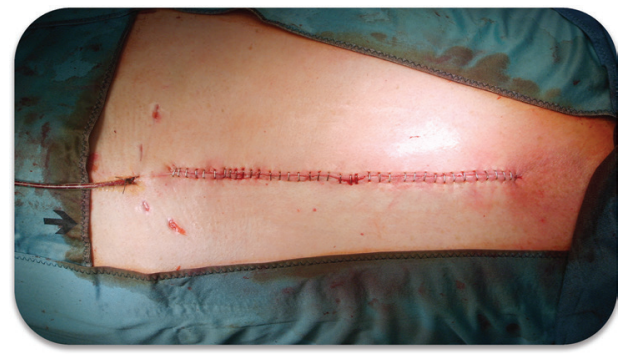

Fig. 2 Debridement of DSWI in a male cardiac surgical patient. (A) Presternal soft tissue VRE infection. (B) Second low-frequency ultrasound-assisted wound debridement session. (C) Surgical debridement of infected scar. (D) Closure of the deep sternal wound after the third low-frequency ultrasound-assisted wound debridement session. DSWI: deep sternal wound infection; VRE: vancomycin-resistant Enterococci

postoperative days (POD) in group A versus $26.6 \pm 20.6$ POD $(p=0.958)$ in group B. Patients' characteristics and SWI risk factors are presented in Table 1. The mean age was $66.6 \pm 10.6$ and $65.4 \pm 9.48$ years $(p=0.691)$ for groups $\mathrm{A}$ and $\mathrm{B}$, respectively.

A variety of important risk factors for DSWI were analyzed in both groups. The use of bilateral internal thoracic arteries was similar in both groups (13\% (A) vs. $14 \%(\mathrm{~B}))$. The mean operation time $(p=0.058)$ and cardiopulmonary bypass time did not differ between both groups $(p=0.279)$. All other demographic characteristics and known DSWI risk factors were similar in both groups. There was no significant difference in the lengths of ICU stays ( $p=0.156)$ between the groups. The mean follow-up time was $7.30 \pm 2.47$ in group $\mathrm{A}$, and $8.72 \pm$ 2.89 months in group B.

\section{Microbiological findings}

Prior to any wound debridement, different varieties of microorganisms and fungi were isolated (Table 2). In group A, 65.2\% (15/23) Gram-positive species were isolated. Among them, $13 \%$ (3/23) were multi-resistant microorganisms. In $17.4 \%(4 / 23)$ of the patient's isolated Gram-negative bacteria, only $4.4 \%(1 / 23)$ was multi-resistant. Microbiological findings in group B did not differ significantly compared to group A. $63.6 \%$
(14/22) Gram-positive species were isolated from DSWI in group B, with $9.1 \%$ multi-resistant Gram-positive; $18.2 \%$ (4/22) Gram-negative and among them $4.5 \%$ $(1 / 22)$ were multi-resistant Gram-negative species. Candida albicans was found in one patient, $4.5 \%(1 / 22)$ in group B.

We performed a quantitative analysis, and divided microbiological findings into four groups according to the intensity of the bacterial contamination (amount of isolated bacteria from the sternal wound): pronounced, moderate, minimal, and negative. Two wound cultures were taken step-by-step before each debridement session in both groups.

After the first debridement session, we found a tremendous bacterial reduction in wound cultures. In group A, 30\% (7/23) of bacteria were isolated versus $63.6 \%(14 / 23)$ in group B $(p=0.037)$. In both groups, the other cultures moderate $(43.5 \%$ (A) vs. $18.2 \%$ (B), $p=0.107)$, minimal (17.4\% (A) vs. $13.6 \%$ (B)) and negative quantity isolated microbiological species were statistically not significant.

After the second debridement session, a reduction of the bacterial contamination was noted in both groups. No pronounced bacterial contamination could be detected in group A after the LF-UAW intervention, compared with $13.6 \%(3 / 22)$ in group B $(p=0.108)$. In other 
Table 1 Patients demographics and DSWI risk factors

\begin{tabular}{lccc}
\hline Parameter & $\begin{array}{c}\text { Group A } \\
(\mathrm{n}=23)\end{array}$ & $\begin{array}{c}\text { Group B } \\
(\mathrm{n}=22)\end{array}$ & $p$ value \\
\hline Mean age (years) & $66.6 \pm 10.6$ & $65.4 \pm 9.48$ & 0.691 \\
BMI (kg/m2) & $28.6 \pm 4.4$ & $28.5 \pm 3.75$ & 0.935 \\
DMID & $8(35 \%)$ & $13(59 \%)$ & 0.139 \\
COPD (GOLD >2) & $12(52.2 \%)$ & $8(36.4 \%)$ & 0.372 \\
Nicotine abuses & $18(78.2 \%)$ & $16(72.7 \%)$ & 0.738 \\
Mean creatinine (mg/dL) & $0.86 \pm 0.23$ & $0.96 \pm 0.36$ & 0.270 \\
PAD & $5(21.7 \%)$ & $6(27.3 \%)$ & 0.738 \\
LVEF & & & \\
$\quad<30 \%$ & $2(8.7 \%)$ & $3(13.6 \%)$ & 0.665 \\
$30-50 \%$ & $12(52.2 \%)$ & $6(27.3 \%)$ & 0.129 \\
$>50 \%$ & $9(39.1 \%)$ & $13(59.1 \%)$ & 0.238 \\
CABG (use of IMA) & $20(87 \%)$ & $19(86.4 \%)$ & 1.000 \\
Combined procedures & $3(13 \%)$ & $3(13.6 \%)$ & 1.000 \\
CPB time (min) & $98.7 \pm 52.5$ & $113.4 \pm 35.4$ & 0.279 \\
Operation time (min) & $196.2 \pm 56.2$ & $230.8 \pm 63.0$ & 0.058 \\
Intubation time (days) & $2.1 \pm 1.5$ & $2.7 \pm 1.3$ & 0.159 \\
ICU stay (days) & $4.0 \pm 3.9$ & $5.7 \pm 4.0$ & 0.156 \\
Time of SWI diagnosis (POD) & $26.9 \pm 18.1$ & $26.6 \pm 20.6$ & 0.958 \\
\hline
\end{tabular}

BMI: body mass index; CABG: coronary artery bypass graft; $\mathrm{CPB}$ : cardiopulmonary bypass time; DMID: diabetes mellitus insulin dependent; COPD: chronic obstructive pulmonary disease; PAD: peripheral artery disease; LVEF: left ventricular ejection fraction; combined procedures: $\mathrm{CABG}$ and valve surgery; POD: postoperative day; ICU: intensive care unit; IMA: internal mammary artery

Table 2 Microbiological findings

\begin{tabular}{lccc}
\hline & $\begin{array}{c}\text { Group A } \\
(\mathrm{n}=23)\end{array}$ & $\begin{array}{c}\text { Group B } \\
(\mathrm{n}=22)\end{array}$ & $p$ value \\
\hline Gram "+" species & $15 / 23(65.2 \%)$ & $14 / 22(63.6 \%)$ & 1.000 \\
Multi-resistant Gram "+" species & $3 / 23(13.0 \%)$ & $2 / 22(9.1 \%)$ & 1.000 \\
Gram "-" species & $4 / 23(17.4 \%)$ & $4 / 22(18.2)$ & 1.000 \\
Multi-resistant Gram "-" species & $1 / 23(4.4 \%)$ & $1 / 22(4.5 \%)$ & 1.000 \\
Candida albicans & 0 & $1 / 22(4.5 \%)$ & 0.488 \\
\hline
\end{tabular}

Gram “+”: Gram-positive microorganism; Gram "-”: Gram-negative microorganism; multi-resistant species includes VRE: vancomycin-resistant Enterococci, MDRGN: multidrug-resistant Gram-negative bacteria; MRSA: methicillin-resistant Staphylococcus aureus; methicillin-resistant Staphylococcus epidermidis

cultures, moderate $(8.7 \%$ (A) vs. $22.7 \%(\mathrm{~B}), p=0.242)$ and minimal $(34.8 \%$ (A) vs. $40.9 \%$ (B), $p=0.763$ ) amounts of microorganism were statistically not different. Negative cultures were detected in $56.5 \%$ of group A versus $22.7 \%$ of group B patients ( $p=0.033$ ). At the same time after the second debridement, the wound in group A appeared macroscopically cleaner and more granulation tissues were also noted compared to group B.

After the third debridement session, we isolated only one positive culture $(4.3 \%)$ with minimal quantity of bacteria in group A and $54.5 \%$ in group B $(p=0.001)$. Furthermore, $13.6 \%$ of the patients in group B still had moderate amounts, while $31.8 \%$ had minimal amounts of bacterial cultures.

The postoperative data are summarized in Table 3 . The mean duration of VAC therapy was $19.9 \pm 4.79$ days in group $\mathrm{B}$ and $7.35 \pm 4.79$ in group $\mathrm{A}(p=0.001)$. The mean antibiotic therapy was $27.4( \pm 17.7)$ days in group B, and $15.48( \pm 6.07)$ days in group A $(p=0.003)$. The length of hospital stays due to SWI was significantly shorter in group A $16.82( \pm 8.55)$ days when compared with group B $34.2( \pm 17.7)$ days $(p=0.0001)$. The incidence of SWI recurrence was $4.3 \%(1 / 23)$ in group A versus $731.8 \%(7 / 22)$ in group $\mathrm{B}(p=0.022)$. The mean 
Table 3 Postoperative data

\begin{tabular}{lccc}
\hline Parameter & $\begin{array}{c}\text { Group A } \\
(\mathrm{n}=23)\end{array}$ & $\begin{array}{c}\text { Group B } \\
(\mathrm{n}=22)\end{array}$ & $p$ value \\
\hline Mean duration of VAC therapy (days) & $7.35 \pm 4.79$ & $19.9 \pm 17.2$ & 0.001 \\
Mean duration of antibiotic therapy (days) & $15.48 \pm 6.07$ & $27.4 \pm 17.7$ & 0.003 \\
Length of hospital stay due to SWI (days) & $16.82( \pm 8.55)$ & $34.2 \pm 17.7$ & 0.0001 \\
Recurrent sternal wound infection & $1 / 23(4.3 \%)$ & $7 / 22(31.8 \%)$ & 0.022 \\
Mean Follow-up time (months) & $7.30( \pm 2.47)$ & $8.72 \pm 2.89$ & 0.078 \\
\hline
\end{tabular}

VAC: vacuum-assisted closure; SWI: sternal wound infection

follow-up time was $7.30 \pm 2.47$ months in group $\mathrm{A}$ and $8.72 \pm 2.89$ in group B $(p=0.078)$.

\section{Discussion}

The aim of this study was to evaluate the clinical outcomes of post-cardiac surgery DSWI, by comparing the outcomes of patients who underwent conventional surgical debridement and VAC with those of patients who received a combined UAW debridement and VAC.

The wound healing process is complex and crucial to maintain the barrier function of skin. Many factors can affect the cascade of events involved in wound healing, resulting in chronic, non-healing wounds, which in turn cause significant discomfort and distress for the patients as well as the deprivation of an enormous amount of medical resources. In 1997, a new era in the treatment of wound infection began, as Argenta and Morykwas ${ }^{9)}$ introduced the application of negative pressure on wounds. The VAC therapy rapidly gained widespread acceptance although the mechanisms responsible for the VAC effect on the healing of wounds are still unclear. A correlation between the intensity of negative pressure and blood flow at the microvascular level has been demonstrated by Wackenfors et al. ${ }^{10)}$ Recently, ultrasound has been used for the treatment of a variety of wounds. Many studies demonstrated that LF-UAW treatment accelerates the healing process. ${ }^{11,12)}$ Ultrasound as a therapeutic agent in chronic wound healing has been extensively studied. The overall acceptance in other medical disciplines (e.g., septic, vascular, and orthopedic surgery) is very good, especially for soft tissue and bone debridement. ${ }^{8,11,12)}$ The application of ultrasound may produce some biophysical effects, which are related to wound healing. These include a shift in cellular protein synthesis and release, blood flow and vascular permeability, and angiogenesis. ${ }^{13,14)}$ Such effects have encouraged clinicians to use therapeutic ultrasound for wound infection treatment. Based on the cavitation effect, the bactericidal properties of ultrasonic lavage offer an effective treatment for infections, and sufficiently eliminate biofilms. ${ }^{15-17)}$ We accordingly observed a faster secondary wound closure time following ultrasound treatment in our study. The properties of ultrasonic debridement allow for a very gentle treatment of chronic wounds, which leads to selective necrosectomy, a reduction in the bacterial burden and stimulation of granulation. Only necrotic tissues, cell remnants, biofilms, and contaminations are safely removed, and vital tissue is hardly affected. ${ }^{8,12,18)}$ However, not all necrotic bone tissue, infected scar, or steel wires can be removed with LF-UAW alone, a selective surgical debridement is still needed. A combination of conventional surgical debridement techniques and ultrasonic lavage is most effective. ${ }^{8)}$ With the LF-UAW, we could achieve a maximal reduction of bacterial contamination of the wound, as no surgical preparation was able to destroy biofilms as effectively as ultrasonic lavage. Despite all noted advantages of LF-UAW, we also documented a few minor disadvantages of the LF-UAW system during tissue contact. ${ }^{1)}$ The tissue temperature rose slightly (mean: $<2^{\circ} \mathrm{C}$ ) during the treatment with the LF-UAW system. Tissue overheating can lead to necrosis. In our patient group, sternal tissue overheating was prevented by continuous saline irrigation. ${ }^{2)}$ The possibility of contamination or transmission of disease by infectious saline mist produced by the transducer in patients with resistant viral infections (e.g., HIV, hepatitis, and methicillinresistant staphylococcus aureus) is unknown. ${ }^{3)}$ The LFUAW system transducer contact to steel wires might lead to a complete defect. ${ }^{4)}$ Not all necrotic tissue can be removed by utilizing the LF-UAW system. For infected scars or devitalized bone, a surgical instrument is still needed. ${ }^{5)}$ For the LF-UAW treatment, general anesthesia is mandatory. ${ }^{6)}$ In DSWI, post-LF-UAW debridement sterile wound closure with VAC is necessary. ${ }^{7)}$ The high costs of the LF-UAW system and the need for transducer sterilization after every session might be an additional 
limitation of the LF-UAW. On the other hand, LF-UAW reduced the duration of antibiotics therapy and the total hospital length of stay significantly compared to DSWI patients, who treated with VAC alone, leading to an overall better cost-effective treatment compared to VAC alone. However, ultrasonic therapy is easy to learn and time-saving, and shows a favorable risk profile. ${ }^{8)}$ In comparison to VAC as the widely used standard treatment of SWI, the use of LF-UAW treatment in our study displays better effectiveness due to destruction of biofilms and bacterial walls. We were able to achieve a faster cleansing of the infected wounds and therefore shorter hospital stays, which in turn had an important effect on patients' psychological stress, as major depression can occur due to postoperative complications, such as SWI and due to longer hospital stays. ${ }^{19)}$ Combined LF-UAW and VAC treatment did facilitate early wound healing, which could result in a reduction of total healthcare costs, and the improvement of patients' quality of life.

\section{Study limitations}

Our study is limited by the usual shortcomings of a small cohort single-center study and a relatively heterogeneous patient group. The fortunately low incidences of DSWI only allow for small patient numbers per center. The small number of patients in this singlecenter approach may limit generalization of the results. With the given sample sizes of $n=23$ (group A) and $\mathrm{n}=22$ (group B), the power to detect an effect size of 0.93 at a significance level of 0.05 is 0.8 (two-sided Mann-Whitney U-test). For dichotomous variables, the power of 0.8 is achieved for proportions $70.5 \%$ versus $20.0 \%$ (group A vs. group B) or $58.8 \%$ versus $7.7 \%$, to give two examples (two-sided Fisher's exact test). Smaller effect sizes are less probable to be detected with the given data set. Given these limitations, the greater mean benefit found for LF-UAW in this study requires further confirmation in larger randomized clinical trials.

\section{Conclusion}

The low-frequency ultrasonic wound debridement system is an advanced technical tool for the treatment of SWI with effective end results. In combination with VAC therapy, it has the potential for more effective wound care in SWI. This combination therapy employed in our study shortened lengths of hospital stays and lessened the use of antibiotics significantly compared to VAC therapy alone, which may lead to even much more costefficient DSWI treatment.

\section{Funding Statement}

This study was supported by a research grant from Söring, Quickborn, Germany. LT received the grant. The sponsor was not involved in any phase of the study preparation, data collection, data analysis, or manuscript preparation.

\section{Disclosure Statement}

All authors have no conflicts of interest to declare.

\section{References}

1) Lemaignen A, Birgand G, Ghodhbane W, et al. Sternal wound infection after cardiac surgery: incidence and risk factors according to clinical presentation. Clin Microbiol Infect 2015; 21: 674.e11-8.

2) Willy C, Engelhardt M, Stichling M, et al. The impact of surgical site occurrences and the role of closed incision negative pressure therapy. Int Wound J 2016; 13: 35-46.

3) Jeffers AM, Maxson PM, Thompson SL, et al. Combined negative pressure wound therapy and ultrasonic MIST therapy for open surgical wounds: a case series. Journal of wound, ostomy, and continence nursing: official publication of The Wound, Ostomy and Continence Nurses Society 2014; 41: 181-6.

4) Percival SL. Importance of biofilm formation in surgical infection. Br J Surg 2017; 104: e85-94.

5) Han G, Ceilley R. Chronic wound healing: a review of current management and treatments. Adv Ther 2017; 34: 599-610.

6) Breuing KH, Bayer L, Neuwalder J, et al. Early experience using low-frequency ultrasound in chronic wounds. Ann Plast Surg 2005; 55: 183-7.

7) Risman BV, Rybal'chenko OV, Bondarenko VM, et al. [Repression of bacterial biofi lms in suppurative necrotic complications of diabetic foot syndrome by ultrasound cavitation]. Zh Mikrobiol Epidemiol Immunobiol 2011; 4: 14-9.

8) Tewarie L, Moza AK, Zayat R, et al. Ultrasoundassisted treatment of sternocutaneous fistula in poststernotomy cardiac surgery patients. European journal of cardio-thoracic surgery: official journal of the European Association for Cardio-thoracic Surgery 2015; 47 : e180-7; discussion e7.

9) Argenta LC, Morykwas MJ. Vacuum-assisted closure: a new method for wound control and treatment: clinical experience. Ann Plast Surg 1997; 38: 563-76; discussion 577. 
10) Wackenfors A, Gustafsson R, Sjögren J, et al. Blood flow responses in the peristernal thoracic wall during vacuum-assisted closure therapy. Ann Thorac Surg 2005; 79: 1724-30; discussion 1730-1.

11) Taradaj J, Franek A, Brzezinska-Wcislo L, et al. The use of therapeutic ultrasound in venous leg ulcers: a randomized, controlled clinical trial. Phlebology 2008; 23: 178-83.

12) Voigt J, Wendelken M, Driver V, et al. Low-frequency ultrasound (20-40 kHz) as an adjunctive therapy for chronic wound healing: a systematic review of the literature and meta-analysis of eight randomized controlled trials. Int J Low Extrem Wounds 2011; 10: 190-9.

13) Poltawski L, Watson T. Transmission of therapeutic ultrasound by wound dressings. Wounds 2007; 19: 1-12.

14) Young SR, Dyson M. The effect of therapeutic ultrasound on angiogenesis. Ultrasound Med Biol 1990; 16: 261-9.
15) Carmen JC, Roeder BL, Nelson JL, et al. Ultrasonically enhanced vancomycin activity against Staphylococcus epidermidis biofilms in vivo. J Biomater Appl 2004; 18: 237-45.

16) Beheshti A, Shafigh Y, Parsa H, et al. Comparison of high-frequency and MIST ultrasound therapy for the healing of venous leg ulcers. Adv Clin Exp Med 2014; 23: 969-75.

17) Qian Z, Sagers RD, Pitt WG. The effect of ultrasonic frequency upon enhanced killing of P. aeruginosa biofilms. Ann Biomed Eng 1997; 25: 69-76.

18) McDonald WS, Nichter LS. Debridement of bacterial and particulate-contaminated wounds. Ann Plast Surg 1994; 33: 142-7.

19) Ghoneim MM, O'Hara MW. Depression and postoperative complications: an overview. BMC Surg 2016; 16: 5. 